\title{
Comprehensive Analysis of circRNA-associated Competing Endogenous RNA Networks and Immune Infiltration in Gastric Cancer
}

beibei $x u$

Wenzhou People's Hospital https://orcid.org/0000-0002-2574-5901

Endian Zheng

Wenzhou People's Hospital

Yi Huang

Wenzhou People's Hospital

Liang Zheng

Wenzhou People's Hospital

Qiaoli Lan

Wenzhou People's Hospital

Xiaolu Zhou

Zhejiang Provincial People's Hospital

Xiaoge Geng

Zhejiang Provincial People's Hospital

Yanan Wang

Zhejiang University of Technology

Xiuyan Wang ( $\sim$ wangxiuyan12@sina.com )

Wenzhou People's Hospital

Wensheng Pan

Zhejiang Provincial People's Hospital

\section{Research Article}

Keywords: Gastric cancer, CircRNA, competitive endogenous RNA, Immune infiltrates

Posted Date: August 19th, 2021

DOI: https://doi.org/10.21203/rs.3.rs-805632/v1

License: @ (i) This work is licensed under a Creative Commons Attribution 4.0 International License. Read Full License 


\section{Abstract}

Background

Circular RNA (circRNA) has been shown to be an important regulator in gastric cancer (GC). However, functions and regulatory mechanisms of circRNA-related competitive endogenous RNA (ceRNA) in GC have not been established.

Methods

CircRNA data and clinical data were downloaded from the GEO and TCGA databases. The ceRNA and ProteinProtein Interaction (PPI) networks were constructed through bioinformatics analysis. Function enrichment analysis was performed. Additionally, correlations between expression levels of the top 10 hub genes and immune cell infiltration levels, histopathological grade and clinical stage were determined to establish their clinical values. The differentially expressed circRNA (DEcircRNA) was validated by quantitative real-time PCR (qRT-PCR).

Results

Screening of the GEO and TCGA databases revealed a total of 1627 DEcircRNAs, 6516 DEmRNAs, and 1451 DEmiRNAs. The ceRNA interaction network contained 2 circRNAs, 3 miRNAs and 55 mRNAs. Meanwhile, Gene Ontology (GO) analysis revealed a total of 323 biological processes (BP) terms, 53 cellular components (CC) terms, 51 molecular functions (MF) terms, while the Kyoto Encyclopedia of Genes and Genomes (KEGG) revealed 4 signaling pathways. Gene Set Enrichment Analysis (GSEA) analysis revealed that EPHA4, NCAM1 and NRXN1 were positively correlated with the axon guidan and adhesion molecules pathways. Most of top 10 hub genes were positively correlated with B cells, CD8+ T cells, CD4+ T cells, Neutrophils and Dendritic Cell infiltration.

Correlation analysis between hub genes and clinical phenotypes revealed that elevated expressions of EPHA4 and KCNA1 indicated poor tissue differentiation and were associated with clinically advanced stages of GC. The qRTPCR results revealed that the expression of has_circ_0002504 was significantly down-regulated in 3 GC cell lines which was consistent with the results of our bioinformatics analysis.

Conclusions

Has_circ_0001998 and has_circ_0002504 are potential diagnostic biomarkers for GC, and the high expressions of both EPHA4 and KCNA1 may predict poor prognosis.

\section{Introduction}

Globally, gastric cancer (GC) is one of the commonest and the most fatal malignant tumors. In 2018, there were about 1.03 million new morbidities and 780,000 mortalities associated with GC, with its morbidity and mortality ranking fifth and third, respectively (Bray et al. 2018). Despite diagnostic and therapeutic advances for GC, the 5year overall survival rate for most patients with advanced GC after treatment is only about 30\% (Katai et al. 2018) (Veisani et al. 2016) (Yang et al. 2020). Since molecular mechanisms of GC pathogenesis and progression have not been clearly elucidated, there is a lack of good early diagnostic biomarkers and therapeutic targets. With advances in bioinformatics analysis, circRNA microarray analysis and high-throughput sequencing technology, studies have indicated that circRNAs play an important role in GC proliferation, apoptosis and metastasis (Li et al. 2019b). Due to their conservative and stable structure, cricRNAs are considered as great promising prognostic as 
well as early diagnostic biomarkers for GC and are potential therapeutic targets (Su et al. 2019) (Sun et al. 2018). However, their specific roles have not been fully established. To further evaluate the molecular mechanisms of circRNAs in GC and to identify better biomarkers for diagnosis or treatment, we predicted target genes by screening differentially expressed circRNAs (DEcircRNAs) and constructing a circRNA-miRNA-mRNA interaction network across multiple databases. Clinical values of these hub-genes were determined through correlation analysis and immune infiltration analysis.

It has been reported that circRNAs can be used as ceRNAs to retain endogenous RNA and are also regulators of related genes expression to intervene in the occurrence and development of many kinds of carcinomas ( $\mathrm{Li}$ et al. 2019b; Liu et al. 2017; Su et al. 2019). Han et al. evaluated the role of circRNAs in GC and its regulatory mechanisms by constructing a ceRNA interaction network, and screened out more than 10 genes, such as SERPINE1, which were identified as possible diagnostic and prognostic indicators for GC (Han et al. 2021). Deng et al. found that CircRHOBTB3 is a ceRNA of miR-654-3p, which inhibits GC progression by activating the p21 signaling pathway (Deng et al. 2020). Li reported that hsa_circ_0017639 promotes GC proliferation and metastasis by sponging miR-224-5p and up-regulating USP3 (Li et al. 2020). Moreover, circPSMC3, had been shown to regulate PTEN expression by sponging miR-296-5p in GC (Rong et al. 2020).

However, since studies on circRNAs is still in their infancy, various limitations exist. For instance, many results are based on bioinformatics analysis, whose research scope is not comprehensive enough and they lack further investigations to confirm their conclusions and exact mechanisms of circRNA in tumorigenesis (Han et al. 2021). Then, small numbers of tissue samples are mostly sourced from the same hospital in most studies, as they should preferably be sourced from different hospitals or even different regions. Moreover, many studies on GC, such as analysis of the correlation between targeted genes and clinical features and immune infiltration are not in-depth.

In this study, we constructed a ceRNA regulatory network composed of 2 circRNA, 3 miRNAs and 55 mRNAs through bioinformatics analysis, studied the correlativity between expression levels of the top 10 hub genes and infiltration levels of immune cells, as well as histopathological and clinical stages. Our findings illuminate the potential regulatory mechanisms of circRNAs in GC development at molecular level, and indicate that circRNAs have the potential as diagnostic and therapeutic biomarkers for GC.

\section{Materials And Methods}

\section{Data pretreatment}

A total of two datasets of circRNA microarray data, GSE83521 and GSE100170, were downloaded from the GEO database. Clinical (phenotype) features, RNAseq data and miRNA data of GC were obtained from the TCGA database using the UCSC Xena browser. To connect different data, we matched, selected and deleted different data for various samples. Samples were grouped according to cancer and the adjacent non-cancerous group, mRNAs with more than $80 \%$ zero expression values were deleted, as well as miRNAs with all zero expression values. In total, mRNA sequencing data for 407 samples (375 cancerous vs 32 adjacent non-cancerous) and miRNA data for 477 samples (436 cancerous vs 41 adjacent non-cancerous) were obtained.

\section{Differentiation analysis}


By comparing the cancer and the adjacent non-cancerous groups, we determined differentially expressed genes (DEGs), including DEcircRNAs, DEmiRNAs and DEmRNAs with thresholds $p<0.05$ and $|\log 2 \mathrm{FC}|>1 \mathrm{using} R$ (Version 3.6.3) package limma (Version 3.42.2) (Ritchie et al. 2015). Then, expression values of DEGs were clustered based on Euclidean distance by pheatmap package (Version 1.0.12) (Tian et al. 2021), and were displayed using heatmaps, volcano plots and PCA.

\section{Construction of ceRNA and PPI networks}

The DEcircRNAs were obtained from the intersection of GSE83521 and GSE100170 datasets. Potential miRNA targets for DEcircRNAs were predicted using the Circlnteractome online tool (https://circinteractome.irp.nia.nih.gov/mirna_target_sites.html) (Dudekula et al. 2016) and compared with the screened-out DEmiRNAs downloaded from the TCGA. Then, downstream DEmiRNAs regulated by DEcircRNAs were filtered out. Subsequently, target mRNAs that are regulated by DEmiRNAs were predicted using the mirwalk online tool (http://mirwalk.umm.uniheidelberg.de/) (Sticht et al. 2018) and compared with the DEmRNAs downloaded from the TCGA. Then, downstream DEmRNAs that are regulated by DEmiRNAs were filtered out. Ultimately, intersection results of DEcircRNAs - DEmiRNAs - DEmRNAs were presented using a Venn diagram by R package VennDiagram (1.6.20) (Lam et al. 2016).

The PPI analysis on DEmRNAs in the ceRNA network was constructed by the STRING database (version: 11.0, http://www.string-db.org/) (Szklarczyk et al. 2019) with a confidence (combined score) of >0.15 as the threshold. The relevant tsv files were downloaded and the PPI network was visualized using the Cytoscape software (Doncheva et al. 2019).

Regulatory networks for unfiltered circRNA-miRNA and filtered miRNA-mRNA were constructed and visualized using cytoscape. Ultimately, the ceRNA network, constructed with DecircRNAs-DEmiRNAs-DEmRNAs, was then visualized.

\section{GO and KEGG Analyses}

We performed the GO and KEGG pathway enrichment analyses of the DEmRNAs using R package ClusterProfiler (Yu et al. 2012) to identify the signaling pathways and biological functions that might be affected by the mRNAs involved in the ceRNA network. The significance threshold was set at $p \leq 0.05$, and results were visualized using a bubble diagram.

\section{GSEA enrichment analysis}

We performed GSEA enrichment analysis on the top 10 hub genes in the PPI network using the R-package clusterProfiler. The GSEA statistical processes included calculating the enrichment fraction, estimating the significance of enrichment fraction and correcting multiple hypothesis testing.

\section{Correlation analysis of immune infiltration}

We used the TIMER (https://cistrome.shinyapps.io/timer/) (Li et al. 2017), a online tool, to analyze and visualize correlations between expression levels of the top 10 hub genes and immune infiltrate levels in GC. By inputting genes and cancer types, the scatter diagram showing the purity-corrected Spelman Rau value and statistical significance was generated. 


\section{Correlations between hub genes and clinical features}

The GC patients were grouped based on clinical features, including age, cancer stage and histological grade among others. Differential expressions of the top 10 genes between each pair of groups were presented using a box diagram.

\section{Valiation of the regulatory role of the ceRNA network in vitro assays}

Human gastric epithelial cell line GES-1 and GC cell lines HGC-27 were obtained from Beyotime (ShangHai, China), GC cell lines AGS were derived from American Tissue Culture Collection (ATCC, Rockville, MD, USA) while MKN-45 were derived from iCell Bioscience Inc, (ShangHai, China). All cells were incubated in RPMI-1640 (GIBCO, Invitrogen Corporation, NY, USA), supplemented with 10\% fetal bovine serum (FBS) (Hyclone, Logan, UT, USA). All cell lines were grown at $37^{\circ} \mathrm{C}$ in $5 \% \mathrm{CO}_{2}$.

Total RNA of cells were extracted by Total RNA extraction kit (Takara, Japan) and reverse transcribed into cDNA using the iScript cDNA synthesis kit (Bio-Rad, Hercules, CA, USA). CircRNA expression level was measured by qRTPCR using SYBR Green Premix Ex TaqTM (Takara, Japan) on ABI 7500 real-time fluorescence quantitative PCR instrument (Applied Biosystems Inc, USA). Primers for circRNAs and GAPDH were as follows: hsa_circ_0001998: F-CCTGGCGTTGGATTATGCTC and R-AAGCAGCAACCGGAGAGATT; hsa_circ_0002504: F-

TTTCGTTTTGATGGCTGGCT and R-TCATCTTTGATGCTGTTGTGCT; GAPDH: F- GCACCGTCAAGGCTGAGAAC and R-TGGTGAAGACGCCAGTGGA.

\section{Statistical analysis}

All bioinformatic analysis was conducted using R Version 3.6.3 software. All data related to qRT-PCR were analyzed with Graphpad 7.0 and indicated as mean \pm SD. The statistical differences between groups were tested by one-way ANOVA and Tukey's test. $P$-value $<0.05$ was considered as statistically significant.

\section{Results}

\section{Identification of DEGs}

A total of 150 DEcircRNAs in the GSE83521 data set (80 up-regulated vs 70 down-regulated; Fig. $1 \mathrm{~A}-\mathrm{C}$ ), and 1477 DEcircRNAs in the GSE100170 data set (497 up-regulated vs 980 down-regulated; Fig. 1D-F) were obtained from the GEO database (supplementary files S1).

Baseline characteristics of GC patients from the TCGA database are presented in Table 1. A total of 29579 expressed genes, including 6516 DEmRNAs (3684 up-regulated vs 2832 down-regulated; Fig. 1G-I) and 1451 expressed miRNAs, including 108 DEmiRNAs (87 up-regulated vs 21 down-regulated; Fig. 1J-L) were screened out (supplementary files S2).

\section{Construction of ceRNA, PPI interaction networks}

Based on the Circlnteractome database, the 2 DEcircRNAs that were obtained at the intersection of the 2 GEO datasets (GSE83521 and GSE100170) (Fig. 2A) predicted 57 miRNA-binding sites (Fig. 2D), which were then intersected with 108 DEmiRNAs from the TCGA database. Three DEmiRNAs were screened out (Fig. 2B). Based on the mirwalk database, the 3 DEmiRNAs predicted 254 target mRNAs, which were then intersected with 6516 
DEmRNAs from the TCGA database. Only 55 DEmRNAs were screened (Fig. 2C), and were then used to construct the DEmiRNA-DEmRNA network (Fig. 2E). Finally, the ceRNA interaction network consisting of the 2 DEcircRNAs, 3 DEmiRNAs and 55 DEmRNAs was constructed (Fig. 2F). Additionally, we constructed the PPI network using the 55 DEmRNAs through the STRING database (Fig. 2G-I) (supplementary files S3).

\section{GOIKEGG analysis}

In order to investigate the functions of DEGs in the ceRNA network, we performed GO and KEGG analysis. A total of 323 BP terms, 53 CC terms, 51 MF terms were enriched in GO analysis. Significantly enriched BP terms were correlated with central nervous system projection neuron axonogenesis, central nervous system neuron axonogenesis, regulation of cation transmembrane transport, regulation of ion transmembrane transport, and central nervous system neuron development. Significantly enriched CC terms were voltage-gated potassium channel complex, potassium channel complex, ion channel complex, synaptic membrane, and transmembrane transporter complex. In the aspect of molecular function (MF), the most enriched terms were voltage-gated ion channel activity, voltage-gated channel activity, ion gated channel activity, potassium channel activity, and gated channel activity (Fig. 3A-C; Table 2; supplementary files S4). KEGG analysis revealed that those DEGs were notably enriched in glycosaminoglycan biosynthesis-chondroitin sulfate/dermatan sulfate, axon guidance, GABAergic synapse and salmonella infection (Fig. 3D; Table 3; supplementary files S4).

\section{GSEA analysis}

We performed GSEA analysis for top 10 hub genes to identify their correlation with with signaling pathways in GC. According to the expression correlation between EPHA4, NCAM1 and NRXN1 and other genes, the most differentially enriched pathway in EPHA4 was axon guidance, while in both NCAM1 and NRXN1, adhesion molecules pathways were enriched (Fig. 3E-G; Table 4; supplementary files S5).

\section{Immune infiltration analysis}

To evaluate the clinical value of these hubgenes in GC, we analyzed the correlation between the top10 hub genes expression and immune infiltrates, including B cells, CD8+ T cells, CD4+ T cells, macrophages, neutrophils and dendritic cells. As shown in Figure 9, the far left represents the expression levels of genes showing tumor purity. Half of the 10 hubgenes were inversely correlated with tumor purity, while the rest were not. DCLK1, DLG2, EPHA4, NCAM1, NRXN1 and SCN2B observably have positive correlation with all the above mentioned 6 immune cells; KCNA1 was positively correlated with CD8+ T cells, CD4+ T cells, macrophages, neutrophils and dendritic cells; KCNIP2 was positively associated with B cells, CD4+ T cells, macrophages and dendritic cells; UNC13A was positively associated with CD4+ T cells, macrophages and dendritic cells; and EPHB2 were negatively associated with CD8+ T cells, neutrophils and dendritic cells (Fig. 4A-J). Among them, EPHA4 and KCNA1 were positively correlated with both macrophage $(R=0.417, p=5.53 E-17$ and $R=0.266, p=1.93 E-07$, respectively) and dendritic cell infiltrations $(R=0.559, p=8.24 E-32$ and $R=0.376, p=6.4 E-14$, respectively). These findings imply that hubgenes involved in our PPI network were strongly correlated with the immune system.

\section{Clinical correlation analysis}

To elucidate on the clinical roles and significance of hub gene expression, a total of $407 \mathrm{GC}$ samples with all patients' characteristics (gender, age, histological grade, clinical stage, etc.) from the TCGA database were analyzed (Table 1). The cohort included 263 (64.6\%) males and 144 (35.4\%) females, among whom there were 
$117(29 \%)$ below the age of 60 years $(<60)$ and $286(71 \%)$ above 60 years $(\geq 60)$. In terms of tumor stage, 59 (15.4\%) patients were stage I, 126 (32.9\%) were stage II, $156(40.7 \%)$ were stage III and 42 (11\%) were stage IV. Regarding histological grading, 10 (2.5\%) patients were G1, 150 (36.8\%) were G2, 238 (58.5\%) were G3 while 9 (2.2\%) were $\mathrm{Gx}$.

Elevated expressions of NCAM1, DLG2, SCN2B and NRXN1 were associated with advanced age for GC patients ( $\triangle$ 60 vs $\geq 60, p=3.22 E-02, p=1.68 E-02, p=8.09 E-03$ and $p=4.54 E-02$, respectively; Fig. $5 A$ ). Elevated expressions of DCLK1, DLG2, EPHA4, KCNA1, NCAM1, NRXN1 and SCN2B were associated with higher histological grades (G1G2 vs G3-Gx, $p=9.84 E-08, p=1.84 E-03, p=1.33 E-03, p=4.67 E-04, p=2.58 E-03, p=5.54 E-06$ and $p=4.96 E-04$, respectively), and were, therefore, correlated with worse histological differentiation. Suppressed expression of EPHB2 was associated with worse histological differentiation (G1-G2 vs G3-Gx, $p=1.89 E-02)$, as shown in Fig. 5B. Furthermore, overexpression of DCLK1 (stage I vs stage II, $p=4.25 \mathrm{E}-05$; stage I vs stage III, $p=1.39 \mathrm{E}-04$; stage I vs stage IV, $p=1.16 \mathrm{E}-02$ ), EPHA4 (stage I vs stage III, $p=3.31 \mathrm{E}-02$ ), UNC13A (stage I vs stage II, $p=3.2 \mathrm{E}-02$; stage I vs stage IV, $p=3.30 \mathrm{E}-02$ ), NRXN1 (stage I vs stage II, $\mathrm{p}=2.01 \mathrm{E}-02$ ), SCN2B (stage I vs stage II, $\mathrm{p}=2.01 \mathrm{E}-03$; stage I vs stage III, $p=4.83 \mathrm{E}-02$ ) and KCNA1 (stage I vs stage II, $p=1.88 \mathrm{E}-02$ ) were correlated with late clinical stages (Fig. $5 \mathrm{C})$, suggesting that GC patients with these genes expression changed were more prone to enter the progressive stage. Thereinto, elevated expression of EPHA4 and KCNA1 may be associated with poor prognosis.

\section{Validation of circRNA expression}

Since has_circ_0002504 was down-regulated in both GSE83521 and GSE100170 databases showing higher consistency(Fig. 6A-B), it was selected for further validation by qRT-PCR in 4 gastric cell lines (GES-1, AGS, MKN45 and HGC-27). The results suggested that the expression of has_circ_0002504 was significantly down-regulated in GC cells (Fig. 6C) which was consistent with the results of our bioinformatics analysis.

\section{Discussion}

Globally, GC is one of the most common malignancies. Its symptoms usually appear in the late stage, which inhibits early diagnosis, and most patients are diagnosed at the advanced stage of the disease (Allemani et al. 2015; Jiang et al. 2018). In various global regions, the case fatality rate for GC is still as high as $75 \%$, which is a leading contributor to the global burden of disability-adjusted life-year (Thrift \& El-Serag 2020). Due to late diagnosis, most GC patients often miss the optimal treatment window. Early effective detection has particularly importantance on reducing the mortality associated with GC. Researches have reported that circRNAs play a considerable role in GC occurrence and progression and are potential biomarkers and therapeutic targets for GC. In this study, we constructed the ceRNA and PPI interaction network using differentially expressed genes (DEGs) and screened out the hub genes. Then, we performed GO and KEGG analysis, as well as GSEA analysis to elucidate on the enrichment pathways and functions of DEGs and hub genes. We also evaluated the relevance of the top 10 hub genes expression levels and immune infiltrates, and explored the correlations between hub genes and histopathological grade, clinical stage and other clinical features of GC patients.

In this study, we constructed the ceRNA interaction network using 2 key circRNAs, 3 key miRNAs, and 55 key mRNAs. In addition, we used the STRING database to establish the PPI interaction network. We found that one of the 2 key circRNAs, has_circ_0001998, function as miR-490-5p sponge to act on the target genes (EPHA4, KCNA1, etc.; Fig. 2D). Yuan reported that expression levels of has_circ_0001998 are up-regulated in colorectal cancer patients, where they play an anti-tumor role by competitively inhibiting the expression of has-miR-490-5p. 
Moreover, there was no obvious correlation between the expression of has_circ_0001998 and the host gene, while its mRNA expressions were markedly relevant to survival time of colorectal cancer patients. These findings suggest that has_circ_0001998 is highly stable and has a prognostic value (Yuan et al. 2019). In addition, it has been reported that has-miR-490-5p expressed at a low level in various tumor tissues while its overexpression participates in regulating tumor proliferation and invasion as a tumor suppressor in GC (Zhang et al. 2019a), bladder cancer (Wu et al. 2019), esophageal squamous cell carcinoma (Li \& Zang 2019), liver cancer (Fang et al. 2018; Yu et al. 2019), pharyngolaryngeal cancer (Abdeyrim et al. 2019), and renal cell carcinoma (Chen et al. 2016) among others. In GC, has-miR-490-5p may be down-regulated by IncRNA LINC02532 (Zhang et al. 2019a) and circNRIP1 (Zhang et al. 2019b) sponge, promoting GC proliferation, migration and invasion. This result is consistent with the findings of our analysis of the GSE83521 database. Has_circ_0002504 had not been previously reported, but our findings revealed that the expression of this circRNA in both data sets was consistent (supplementary files S9) and was valeted in vitro, suggesting that it may be a relatively new and more specific biomarker.

To further evaluate the 55 target mRNAs that are regulated by the 2 DEcircRNAs in the ceRNA network, GO and KEGG were performed for enrichment analysis. GO analysis revealed that among the BP categories, top five enrichment categories were central nervous system projection neuron axonogenesis, central nervous system neuron axonogenesis, regulation of cation transmembrane transport, regulation of ion transmembrane transport, and central nervous system neuron development. Among the CC categories, the top five categories were voltagegated potassium channel complex, potassium channel complex, ion channel complex, synaptic membrane, transmembrane transporter complex. The top five in the MF category were voltage-gated ion channel activity, voltage-gated channel activity, ion gated channel activity, potassium channel activity, and gated channel activity. Various pathways were enriched in ion channels in GO analysis. Previous studies reported that GC occurrence is correlated with abnormal functions of cross-cell membrane ion channels. Golgi anti-apoptotic protein (GAAP), a multi-transmembrane protein, can form cation-selective ion channels and mediate cell movement as well as adhesion, while the typical signs of malignant differentiation are the changes in migration and adhesion abilities of cancer cells to other cells or extracellular matrix (Carrara et al. 2017). Currently, overexpression of human GAAP (hGAAP) has been reported in some human malignant tissues. The expression of hGAAP between lung cancer tissues and para cancer tissues was significantly different, suggesting that it may be involved in lung carcinogenesis, and is therefore, a new prognostic biomarker for lung cancer patients who never smoked (Wu et al. 2013). It has also been reported that overexpressed hGAAP mRNA in glioblastoma multiforme tumors is related to poor outcomes (Chen et al. 2014). In osteosarcoma and cervical cancer cells, enhanced cell adhesion and migration could regulate local activation of calcium-dependent calpain (Carrara et al. 2015; Saraiva et al. 2013). KCNE2 is a potassium channel protein which is mostly expressed in the cytoplasm of gastric parietal cells. In animal models, suppressed KCNE2 levels were associated significantly low proton secretion, abnormal parietal cell morphologies, acidosis and hypergastrinemia. Functionally, KCNE2 also inhibits cell growth by downregulating CyclinD1, and has an anti-proliferative effect on GC cells (Kundu et al. 2008). Elucidation of these pathways may help clarify GC pathogenesis and hopefully provide prognostic indicators and therapeutic targets for GC. In KEGG analysis, the major enriched pathways were Glycosaminoglycan biosynthesis-chondroitin sulfate/dermatan sulfate, axon guidance, GABAergic synapse, and salmonella infection. Nerve invasion, which is correlated with postoperative tumor recurrence, cancer pain and poor prognosis, is one of the important ways through which GC locally spreads (Bilici et al. 2010). Netrin-1 (NTN1), an axon guiding molecule (Lai Wing Sun et al. 2011), has been shown to be highly expressed in GC, and its expression is associated with nerve invasion and 
lymphatic metastasis. It had been reported that NTN1 plays a key vital in regulating GC invasion in vivo and in vitro (Yin et al. 2019). Another axon guiding molecule, semaphoring 5A, is involved in GC metastasis (Pan et al. 2009), the specific mechanisms may be through MEK/ ERKS pathway activations and the subsequent MMP9trans-activation (Pan et al. 2013). Salmonella infection, through its role in 'inflammatory stimulation' is associated with gallbladder cancer (lyer et al. 2016). Since inflammatory stimuli are associated with tumor development (Sheweita \& Alsamghan 2020), we found that salmonella infection is involved in GC occurrence.

GSEA analysis of hub genes revealed that EPHA4 was positively correlated with axon guidan pathway, while both NCAM1 and NRXN1 were positively correlated with adhesion molecules cams pathway. Semaphorin proteins are a class of axon guiding molecules that play key roles in the development of central nervous system. Their expression is closely correlated with invasion and metastasis for certain human carcinomas (Muratori \& Tamagnone 2012). In oral squamous cell carcinoma, semaphorin 4D can cooperate with Vascular endothelial growth factor (VEGF) to induce cancer growth and angiogenesis (Zhou et al. 2012). Semaphorin 6B has been associated with GC differentiation and metastasis in vivo, as well as tumor cells migration, adhesion and invasion in vitro. These findings suggest that semaphorin $6 \mathrm{~B}$ may play a part in GC occurrence and progression and is a potential biomarker for GC diagnosis, assessment and targeting gene therapy (Ge et al. 2013). Studies have also shown that ectopic expression of semaphorin $3 \mathrm{E}$ inhibits proliferation and colony formation of GC in vitro and of xenografts in vivo. However, its high expression lever inhibited migration and invasion in vitro, implying that downregulated semaphorin $3 \mathrm{E}$ is involved in GC pathogenesis (Chen et al. 2015). Cell adhesion molecules are important in carcinogenesis. L1 cell adhesion molecule (L1CAM) was over expressed in GC tissue and was significantly relevant to poor outcome. Overexpressed L1CAM is an independent prognostic factor for overall and event-free survival, as well as an independent risk factor for distant metastasis of GC, while low levels of L1CAM inhibit proliferation, cell cycle progression, invasion, migration and antinestin apoptosis of GC cells, and suppresses peritoneal metastasis (Ichikawa et al. 2019). Overexpression of the epithelial cell adhesion molecule (EPCAM) is corelated with larger size of tumor, lymphatic metastasis and worse outcomes in GC and might be helpful in prognostic assessment (Dai et al. 2017). Taken together, both pathways are highly associated with GC progression.

Immune cells in the tumor microenvironment are involved in maintaining the homeostasis and tolerance of immune (Li et al. 2019a; Liu et al. 2018; Zeng et al. 2019). Therefore, we evaluated the correlation between the top10 hub genes and immune infiltrates. Most of the 10 hub genes were positively associated with immune infiltrates levels (Fig. 4). Among them, EPHA4 and KCNA1 were positively correlated with macrophage and dendritic cell infiltration. Macrophages infiltrating into tumor tissues, also known as tumor-associated macrophages (TAMs), promote tumor growth by secreting various factors, interacting with other matrix components, promoting angiogenesis and rebuilding the tumor microenvironment (Lee et al. 2014; Serizawa et al. 2016; Song et al. 2019; Veremeyko et al. 2018; Zhao et al. 2016). The degree of macrophage infiltration was positively associated with poor outcome of various carcinomas (Oya et al. 2020). Additionally, M2 polarized TAMs are potential independent predictors for GC prognosis (Zhang et al. 2014), therefore, targeted phenotypic or function specific macrophage subsets may be promising targets for postoperative adjuvant therapy in GC patients (Lin et al. 2019; Zhang et al. 2014). Dendritic cells (DCs) infiltrate various tumor tissues, and the presence of infiltrating DCs in ovarian cancer (Labidi-Galy et al. 2012), breast cancer (Faget et al. 2013) and GC (Liu et al. 2020) indicates a poor prognosis. Li et al. reported that malignant tumor cells and tumor-related pDC secrete indoleamine 2-dioxygenase, a powerful promoter activated by Treg, which may lead to non-responses and thus 
allow malignant cells to escape immune surveillance (Li et al. 2019a). These results suggest that EPHA4 and KCNA1 might play an considerable role in GC immune infiltration and indicate a poor outcome.

To identify the clinical values of these hub genes, we performed a clinical correlation study. Overexpressions of NCAM1, SCN2B and NRXN1 were correlated with advanced ages of GC patients. Overexpressions of NCAM1, SCN2B and NRXN1 were correlated with higher histological grades, implying worse histological differentiation. Suppressed expression levels of EPHB2 were associated with worse histological differentiation. Furthermore, elevated expressions of EPHA4, UNC13A, NRXN1, SCN2B and KCNA1 were correlated with late GC clinical stages. Elevated expression levels of EPHA4 and KCNA1 indicated poor tissue differentiation and were correlated with clinically advanced GC stages. These two genes are potential biomarkers for poor prognosis of GC. MarikoOki et al. found that EphA4 mRNA was highly expressed in GC cells and tissues, and at the protein level, overexpression of EphA4 was markablely correlated with tumor invasion and relapse. Moreover, overall survival time of EphA4 positive cancer patients was observably shorter than that of EphA4 negative cancer patients (Oki et al. 2008). It has also been reported that overexpressed EphA4 is significantly associated with GC recurrence; its high expression level is an independent exist as a predictor for recurrence-free survival and is associated with low survival outcomes (Inokuchi et al. 2018). These conclusions support our postulate, implying that EphA4 plays an important part in GC progression and is a predictor for poor prognosis. Moreover, the mechanisms of KCNA1 in tumors have not been clearly defined. Some studies report that KCNA1 is an overexpressed carcinogene which promotes tumor progression by regulating Wnt, Hhg and Notch signaling pathways as well as mitochondrial ability in cervical cancer (Liu et al. 2018). Lallet-Daher et al. reported that KCNA1, as a voltage-gated delayed potassium channel, can block the transformation induced by oncogenes in NIHT3T3 cells without inducing cell senescence. In addition, they found that down-regulation of KCNA1 enhances the invasiveness of primary breast tumors, suggesting that KCNA1 has tumor inhibitory functions (Lallet-Daher et al. 2013). We proposed for the first time that KCNA1 may be a potential gene in promoting the progression of GC, suggesting a poor prognosis. However, the role of KCNA1 in GC has not been reported yet, and our findings should be further confirmed by more in-depth in vitro and in vivo studies.

This study is corelated with some limitations. First, although microarray-based bioinformatics analysis is a powerful tool for effective evaluation of molecular mechanisms as well as for identifying potential GC biomarkers, a whole loss-of-function and gain-on-function study in vivo and in vitro is needed to prove the expression of DEGs and Hub genes and clarify their functions in GC. Second, we used two microarray analyses, and the data sets were relatively small, which may lead to high false positive rates and ex parte results. To determine the diagnostic accuracy of GC-related circRNAs, multiple data sets should be integrated and the sample size increased. Finally, to confirm the correlations between hub genes and clinical phenotypes of GC, more GC patients should be included in subgroup analysis.

In summary, we constructed the ceRNA interaction and PPI network of GC circRNAs, and analyzed pathway enrichments of DEGs and hub genes. We also determined the correlation between expressions of hub genes and immune infiltrates in GC, as well as its correlation with clinical characteristics. Besides, we valeted the expression of has_circ_0002504 in vitro. Our findings elucidate on the regulatory mechanisms of GC from the perspective of circRNAs. Two circRNAs were found as candidate biomarkers for diagnostic or possible therapeutic targets, while 2 hub genes were found to be novel prognostic factors. It will provide beneficial insights to study on circRNAs and generate novel hypotheses with regards to cancer pathogenesis. However, since our results are based on bioinformatics analysis and in vitro experiments, more studies should confirm these conclusions.

Page $10 / 27$ 


\section{Abbreviations|}

BP biological processes

CC cellular components

cDNA DNA complementary to RNA

ceRNA competing endogenous RNA

circRNA circular RNA

DCs Dendritic cells

DEcircRNA differentially expressed circRNA

DEGs differentially expressed genes

DEmiRNA differentially expressed miRNA

DEmRNA differentially expressed Mrna

EpCAM epithelial cell adhesion molecule

FBS fetal bovine serum

GAAP Golgi anti-apoptotic protein

GC gastric cancer

GEO Gene Expression Omnibus

GO Gene Ontology

GSEA Gene Set Enrichment Analysis

hGAAP human Golgi anti-apoptotic protein

KEGG Kyoto Encyclopedia of Genes and Genomes

L1CAM L1 cell adhesion molecule

MF molecular functions

miRNA micro RNA

NTN1 Netrin-1

PPI Protein-Protein Interaction

qRT-PCR quantitative real-time PCR 
TAMs tumor-associated macrophages

TCGA The Cancer Genome Atlas

VEGF Vascular endothelial growth factor

\section{Declarations}

\section{Ethical approval}

This study does not involve any research on human participants or animals conducted by any of the authors.

\section{Consent for publication}

Not applicable

\section{Availability of data and materials}

All data generated or analysed during this study are included in this published article (and its supplementary information files).

\section{Competing Interests}

The authors declare that they have no competing interests.

\section{Funding}

This work was supported by the Zhejiang medicine key scientific and technology project (grant number: 2018258924).

\section{Author contributions}

$B X, E Z, L Z, X W$ and WP conveived and supervised the project. BX, YH, QL, YW and XW performed the experiments. $X Z, X G, Y W$ and $X W$ performed the statistical analyses and prepared figures and tables. BX, XW and WP drafted the manuscript. All authors critically reviewed and revised the drafts and approved the fnal manuscript.

\section{Acknowledgements}

We thank Dr. Huimei Wang (Helix Medical Institute, shanghai) for guidance on the writing of this manuscript.

\section{References}

Abdeyrim A, Cheng X, Lian M, et al. miR-490-5p regulates the proliferation, migration, invasion and epithelial-mesenchymal transition of pharyngolaryngeal cancer cells by targeting mitogen-activated protein kinase kinasekinase 9. Int J Mol Med. 2019.44(1):240-252. Doi: 10.3892/ijmm.2019.4196

Allemani C, Weir HK, Carreira H, et al. Global surveillance of cancer survival 1995-2009: analysis of individual data for 25676887 patients from 279 population-based registries in 67 countries (CONCORD-2). The Lancet. 2015.385:977-1010. Doi: 10.1016/s0140-6736(14)62038-9 
Bilici A, Seker M, Ustaalioglu BB, et al. Prognostic significance of perineural invasion in patients with gastric cancer who underwent curative resection. Ann Surg Oncol. 2010.17:2037-2044. Doi: 10.1245/s10434-010-1027-y

Bray F, Ferlay J, Soerjomataram I, et al. Global cancer statistics 2018: GLOBOCAN estimates of incidence and mortality worldwide for 36 cancers in 185 countries. CA Cancer J Clin. 2018.68(6):394-424. Doi:

$10.3322 /$ caac. 21492

Carrara G, Parsons M, Saraiva N, et al. Golgi anti-apoptotic protein: a tale of camels, calcium, channels and cancer. Open Biology 2017.7(5):170045. Doi: 10.1098/rsob.170045

Carrara G, Saraiva N, Parsons M, et al. Golgi anti-apoptotic proteins are highly conserved ion channels that affect apoptosis and cell migration. J Biol Chem 2015.290(18):11785-11801. Doi: 10.1074/jbc.M115.637306

Chen H, Xie G, Wang W, et al. Epigenetically downregulated Semaphorin 3E contributes to gastric cancer. Oncotarget. 2015.6(24):20449-20465. Doi: 10.18632/oncotarget.3936

Chen K, Zeng J, Tang K, et al. miR-490-5p suppresses tumour growth in renal cell carcinoma through targeting PIK3CA. Biol Cell. 2016.108(2):41-50. Doi: 108:41-50. Doi: 10.1111/boc.201500033

Chen QR, Hu Y, Yan C, et al. Systematic genetic analysis identifies Cis-eQTL target genes associated with glioblastoma patient survival. PLoS One 2014. 9:e105393. Doi: 10.1371/journal.pone.0105393

Dai M, Yuan F, Fu C, et al. Relationship between epithelial cell adhesion molecule (EpCAM) overexpression and gastric cancer patients: A systematic review and meta-analysis. PLoS One 2017.12(4):e0175357. Doi:

10.1371/journal.pone.0175357

Deng G, Mou T, He J, et al. Circular RNA circRHOBTB3 acts as a sponge for miR-654-3p inhibiting gastric cancer growth. J Exp Clin Cancer Res 2020.39(1):1. Doi: 10.1186/s13046-019-1487-2

Doncheva N, Morris J, Gorodkin J, et al. Cytoscape StringApp: Network Analysis and Visualization of Proteomics Data. J Proteome Res 2019.18(2):623-632. Doi: 10.1021/acs.jproteome.8b00702

Dudekula DB, Panda AC, Grammatikakis I, et al. Circlnteractome: A web tool for exploring circular RNAs and their interacting proteins and microRNAs. RNA Biol 2016.13(1):34-42. Doi: 10.1080/15476286.2015.1128065

Faget J, Sisirak V, Blay JY, et al. ICOS is associated with poor prognosis in breast cancer as it promotes the amplification of immunosuppressive CD4(+) T cells by plasmacytoid dendritic cells. Oncoimmunology 2013.2:e23185. Doi: 10.4161/onci.23185

Fang Z, Li M, Zhang Y, et al. MiR-490-5p inhibits the metastasis of hepatocellular carcinoma by down-regulating E2F2 and ECT2. J Cell Biochem 2018.119:8317-8324. Doi: 10.1002/jcb.26876

Ge C, Li Q, Wang L, et al. The role of axon guidance factor semaphorin 6B in the invasion and metastasis of gastric cancer. J Int Med Res 2013.41:284-292. Doi: 10.1177/0300060513476436

Han T, Chen Z, Chen W, et al. The prognostic value of circular RNA regulatory genes in competitive endogenous RNA network in gastric cancer. Cancer Gene Therapy. 2021.Jan 29. Doi: 10.1038/s41417-020-00270-9 
Ichikawa T, Okugawa Y, Toiyama Y, et al. Clinical significance and biological role of L1 cell adhesion molecule in gastric cancer. British Journal of Cancer 2019.121:1058-1068. Doi: 10.1038/s41416-019-0646-8

Inokuchi M, Nakagawa M, Baogok N, et al. Prognostic Significance of High EphA1-4 Expression Levels in Locally Advanced Gastric Cancer. Anticancer Res 2018.38:1685-1693. Doi: 10.21873/anticanres.12402

lyer P, Barreto SG, Sahoo B, et al. Non-typhoidal Salmonella DNA traces in gallbladder cancer. Infectious Agents and Cancer 2016.11:12. Doi: 10.1186/s13027-016-0057-x

Jiang Y, Zhang Q, Hu Y, et al. ImmunoScore Signature: A Prognostic and Predictive Tool in Gastric Cancer. Ann Surg 2018.267:504-513. Doi: 10.1097/SLA.0000000000002116

Katai H, Ishikawa T, Akazawa K, et al. Five-year survival analysis of surgically resected gastric cancer cases in Japan: a retrospective analysis of more than 100,000 patients from the nationwide registry of the Japanese Gastric Cancer Association (2001-2007). Gastric Cancer 2018.21:144-154. Doi: 10.1007/s10120-017-0716-7

Kundu P, Ciobotaru A, Foroughi S, et al. Hormonal regulation of cardiac KCNE2 gene expression. Mol Cell Endocrinol 2008.292:50-62. Doi: 10.1016/j.mce.2008.06.003

Labidi-Galy SI, Treilleux I, Goddard-Leon S, et al. Plasmacytoid dendritic cells infiltrating ovarian cancer are associated with poor prognosis. Oncoimmunology 2012.1:380-382. Doi: 10.4161/onci.18801

Lai Wing Sun K, Correia JP, and Kennedy TE. Netrins: versatile extracellular cues with diverse functions. Development 2011.138:2153-2169. Doi: 10.1242/dev.044529

Lallet-Daher H, Wiel C, Gitenay D, et al. Potassium channel KCNA1 modulates oncogene-induced senescence and transformation. Cancer Res 2013.73:5253-5265. Doi: 10.1158/0008-5472.CAN-12-3690

Lam F, Lalansingh C, Babaran H, et al. VennDiagramWeb: a web application for the generation of highly customizable Venn and Euler diagrams. BMC Bioinformatics 2016.17:401. Doi: 10.1186/s12859-016-1281-5

Lee $\mathrm{K}$, Hwang $\mathrm{H}$, and Nam KT. Immune response and the tumor microenvironment: how they communicate to regulate gastric cancer. Gut Liver 2014.8:131-139. Doi: 10.5009/gnl.2014.8.2.131

Li B, Jin M, Cao F, et al. Hsa_circ_0017639 expression promotes gastric cancer proliferation and metastasis by sponging miR-224-5p and upregulating USP3. Gene 2020.750:144753. Doi: 10.1016/j.gene.2020.144753

Li F, Sun Y, Huang J, et al. CD4/CD8 + T cells, DC subsets, Foxp3, and IDO expression are predictive indictors of gastric cancer prognosis. Cancer Med 2019.8:7330-7344. Doi: 10.1002/cam4.2596

Li F, and Zang W. Knockdown of IncRNAXLOC_001659 inhibits proliferation and invasion of esophageal squamous cell carcinoma cells. World J Gastroenterol 2019.25:6299-6310. Doi: 10.3748/wjg.v25.i42.6299

Li T, Fan J, Wang B, et al. TIMER: A Web Server for Comprehensive Analysis of Tumor-Infiltrating Immune Cells. Cancer Res 2017.77:e108-e110. Doi: 10.1158/0008-5472.Can-17-0307

Li Z, Ruan Y, Zhang H, et al. Tumor-suppressive circular RNAs: Mechanisms underlying their suppression of tumor occurrence and use as therapeutic targets. Cancer Sci 2019.110:3630-3638. Doi: 10.1111/cas.14211

Page $14 / 27$ 
Lin $\mathrm{C}, \mathrm{He} \mathrm{H}$, Liu H, et al. Tumour-associated macrophages-derived CXCL8 determines immune evasion through autonomous PD-L1 expression in gastric cancer. Gut 2019.68:1764-1773. Doi: 10.1136/gutjnl-2018-316324

Liu J, Liu T, Wang X, et al. Circles reshaping the RNA world: from waste to treasure. Mol Cancer 2017.16:58. Doi: 10.1186/s12943-017-0630-y

Liu W, Zhao J, Li Q, et al. Gastric cancer patients have elevated plasmacytoid and CD1c(+) dendritic cells in the peripheral blood. Oncol Lett 2018.15:5087-5092. Doi: 10.3892/ol.2018.7990

Liu X, Cao Y, Li R, et al. Poor clinical outcomes of intratumoral dendritic cell-specific intercellular adhesion molecule 3-grabbing non-integrin-positive macrophages associated with immune evasion in gastric cancer. Eur $J$ Cancer 2020.128:27-37. Doi: 10.1016/j.ejca.2020.01.002

Muratori C, and Tamagnone L. Semaphorin Signals Tweaking the Tumor Microenvironment. Adv Cancer Res 2012.114:59-85. Doi: 10.1016/B978-0-12-386503-8.00003-X

Oki M, Yamamoto $\mathrm{H}$, Taniguchi $\mathrm{H}$, et al. Overexpression of the receptor tyrosine kinase EphA4 in human gastric cancers. World J Gastroenterol 2008.14:5650-5656. Doi: 10.3748/wjg.14.5650

Oya Y, Hayakawa Y, and Koike K. Tumor microenvironment in gastric cancers. Cancer Sci 2020.111:2696-2707. Doi: $10.1111 /$ cas. 14521

Pan G, Zhang X, Ren J, et al. Semaphorin 5A, an axon guidance molecule, enhances the invasion and metastasis of human gastric cancer through activation of MMP9. Pathol Oncol Res 2013.19:11-18. Doi: 10.1007/s12253012-9550-8

Pan GQ, Ren HZ, Zhang SF, et al. Expression of semaphorin 5A and its receptor plexin B3 contributes to invasion and metastasis of gastric carcinoma. World J Gastroentero/ 2009.15:2800-2804. Doi: 10.3748/wjg.15.2800

Ritchie M, Phipson B, Wu D, et al. limma powers differential expression analyses for RNA-sequencing and microarray studies. Nucleic Acids Res 2015. 43:e47. Doi: 10.1093/nar/gkv007

Rong D, Lu C, Zhang B, et al. Correction to: CircPSMC3 suppresses the proliferation and metastasis of gastric cancer by acting as a competitive endogenous RNA through sponging miR-296-5p. Mol Cancer 2020.19:140. Doi: 19:140. 10.1186/s12943-020-01252-z

Saraiva N, Prole DL, Carrara G, et al. hGAAP promotes cell adhesion and migration via the stimulation of storeoperated Ca2+ entry and calpain 2. Journal of Cell Biology 2013.202:699-713. Doi: 10.1083/jcb.201301016

Serizawa T, Hirata Y, Hayakawa Y, et al. Gastric Metaplasia Induced by Helicobacter pylori Is Associated with Enhanced SOX9 Expression via Interleukin-1 Signaling. Infection and Immunity 2015.84:562-572. Doi:

10.1128/iai.01437-15

Sheweita SA, and Alsamghan AS. Molecular Mechanisms Contributing Bacterial Infections to the Incidence of Various Types of Cancer. Mediators Inflamm 2020:4070419. Doi: 10.1155/2020/4070419

Song H, Wang T, Tian L, et al. Macrophages on the Peritoneum are involved in Gastric Cancer Peritoneal Metastasis. J Cancer 2019.10:5377-5387. Doi: 10.7150/jca.31787

Page $15 / 27$ 
Sticht C, De La Torre C, et al. miRWalk: An online resource for prediction of microRNA binding sites. PLoS One 2018.13:e0206239. Doi: 10.1371/journal.pone.0206239

Su M, Xiao Y, Ma J, et al. Circular RNAs in Cancer: emerging functions in hallmarks, stemness, resistance and roles as potential biomarkers. Mol Cancer 2019.18:90. Doi: 10.1186/s12943-019-1002-6

Sun B, Shi L, Shi Q, et al. Circular RNAs are abundantly expressed and upregulated during repair of the damaged endometrium by Wharton's jelly-derived mesenchymal stem cells. Stem Cell Res Ther 2018.9:314. Doi: $10.1186 / \mathrm{s} 13287-018-1046-3$

Szklarczyk D, Gable A, Lyon D, et al. STRING v11: protein-protein association networks with increased coverage, supporting functional discovery in genome-wide experimental datasets. Nucleic Acids Res 2019.47:D607-D613. Doi: 10.1093/nar/gky1131

Thrift AP, and El-Serag HB. Burden of Gastric Cancer. Clin Gastroenterol Hepatol 2020.18:534-542. Doi: 10.1016/j.cgh.2019.07.045

Tian X, Liu B, Chen L, et al. RNA-Seq Identifies Marked Th17 Cell Activation and Altered CFTR Expression in Different Atopic Dermatitis Subtypes in Chinese Han Populations. Front Immunol 2021.12:628512. Doi: 10.3389/fimmu.2021.628512

Veisani Y, Delpisheh A. Survival rate of gastric cancer in Iran; a systematic review and metaanalysis. Gastroenterol Hepatol Bed Bench 2016.9:78-86.

Veremeyko T, Yung AWY, Anthony DC, et al. Early Growth Response Gene-2 Is Essential for M1 and M2 Macrophage Activation and Plasticity by Modulation of the Transcription Factor CEBPbeta. Front Immunol 2018.9:2515. Doi: 10.3389/fimmu.2018.02515

Wu L, Zhang M, Qi L, et al. ERa-mediated alterations in circ_0023642 and miR-490-5p signaling suppress bladder cancer invasion. Cell Death Dis 2019.10:635. Doi: 10.1038/s41419-019-1827-3

Wu X, Wang L, Ye Y, et al. Genome-wide association study of genetic predictors of overall survival for non-small cell lung cancer in never smokers. Cancer Res 2013.73:4028-4038. Doi: 10.1158/0008-5472.Can-12-4033

Yang L, Ying X, Liu S, et al. Gastric cancer: Epidemiology, risk factors and prevention strategies. Chin J Cancer Res 2020.32:695-704. Doi: 10.21147/j.issn.1000-9604.2020.06.03

Yin K, Wang L, Xia Y, et al. Netrin-1 promotes cell neural invasion in gastric cancer via its receptor neogenin. $J$ Cancer 2019.10:3197-3207. Doi: 10.7150/jca.30230

Yu G, Wang L, Han Y, et al. clusterProfiler: an R package for comparing biological themes among gene clusters. OMICS 2012.16:284-287. Doi: 10.1089/omi.2011.0118

Yu Y, Cai O, Wu P, et al. MiR-490-5p inhibits the stemness of hepatocellular carcinoma cells by targeting ECT2. J Cell Biochem 2019.120:967-976. Doi: 10.1002/jcb.27459

Yuan W, Peng S, Wang J, et al. Identification and characterization of circRNAs as competing endogenous RNAs for miRNA-mRNA in colorectal cancer. PeerJ 2019.7:e7602. Doi: 10.7717/peerj.7602 
Zeng D, Li M, Zhou R, et al. Tumor Microenvironment Characterization in Gastric Cancer Identifies Prognostic and Immunotherapeutically Relevant Gene Signatures. Cancer Immunol Res 2019.7:737-750. Doi: 10.1158/23266066.CIR-18-0436

Zhang C, Ma M-H, Liang Y, et al. Novel long non-coding RNA LINC02532 promotes gastric cancer cell proliferation, migration, and invasion in vitro. World Journal of Gastrointestinal Oncology 2019.11:91-101. Doi:

10.4251/wjgo.v11.i2.91

Zhang $\mathrm{H}$, Wang X, Shen Z, et al. Infiltration of diametrically polarized macrophages predicts overall survival of patients with gastric cancer after surgical resection. Gastric Cancer 2015.18:740-750. Doi: 10.1007/s10120-0140422-7

Zhang X, Wang S, Wang H, et al. Circular RNA circNRIP1 acts as a microRNA-149-5p sponge to promote gastric cancer progression via the AKT1/mTOR pathway. Mol Cancer 2019.18:20. Doi: 10.1186/s12943-018-0935-5

Zhao G, Liu L, Peek RM, et al. Activation of Epidermal Growth Factor Receptor in Macrophages Mediates Feedback Inhibition of M2 Polarization and Gastrointestinal Tumor Cell Growth. J Biol Chem 2016.291:2046220472. Doi: 10.1074/jbc.M116.750182

Zhou H, Yang YH, Binmadi NO, et al. The hypoxia-inducible factor-responsive proteins semaphorin 4D and vascular endothelial growth factor promote tumor growth and angiogenesis in oral squamous cell carcinoma. Exp Cell Res 2012.318:1685-1698. Doi: 10.1016/j.yexcr.2012.04.019

\section{Tables}

Table 1 Baseline data of GC Patients from the TCGA database 


\begin{tabular}{|c|c|c|}
\hline characters & level & overall \\
\hline samples & & 407 \\
\hline \multirow[t]{2}{*}{ Vital states (\%) } & Alived & $247(60.8)$ \\
\hline & Dead & 159(39.2) \\
\hline \multirow[t]{4}{*}{ tumor stage (\%) } & stage I & $59(15.4)$ \\
\hline & stage II & $126(32.9)$ \\
\hline & stage III & $156(40.7)$ \\
\hline & stage IV & $42(11)$ \\
\hline \multirow[t]{4}{*}{ histologic grade (\%) } & G1 & $10(2.5)$ \\
\hline & G2 & $150(36.8)$ \\
\hline & G3 & $238(58.5)$ \\
\hline & GX & $9(2.2)$ \\
\hline \multirow[t]{2}{*}{ Age (\%) } & $<60$ & $117(29)$ \\
\hline & $>=60$ & $286(71)$ \\
\hline \multirow[t]{2}{*}{ Gender (\%) } & male & $263(64.6)$ \\
\hline & female & $144(35.4)$ \\
\hline \multirow[t]{2}{*}{ cancer status (\%) } & TUMOR FREE & $185(71.4)$ \\
\hline & WITH TUMOR & $74(28.6)$ \\
\hline \multirow[t]{4}{*}{ Primary therapy outcome (\%) } & PD & $37(12.8)$ \\
\hline & SD & $30(10.3)$ \\
\hline & PR & $4(1.4)$ \\
\hline & $\mathrm{CR}$ & $219(75.5)$ \\
\hline
\end{tabular}

Table 2 GO enrichment results 


\begin{tabular}{|c|c|c|c|}
\hline ID & Type & Description & pvalue \\
\hline GO:0021952 & $\mathrm{BP}$ & central nervous system projection neuron axonogenesis & 7.00E-07 \\
\hline GO:0021955 & $\mathrm{BP}$ & central nervous system neuron axonogenesis & 2.84E-06 \\
\hline GO:1904062 & $\mathrm{BP}$ & regulation of cation transmembrane transport & 4.86E-05 \\
\hline Go:0034765 & $\mathrm{BP}$ & regulation of ion transmembrane transport & $6.04 \mathrm{E}-05$ \\
\hline GO:0021954 & $\mathrm{BP}$ & central nervous system neuron development & 7.07E-05 \\
\hline GO:0032412 & $\mathrm{BP}$ & regulation of ion transmembrane transporter activity & $9.13 \mathrm{E}-05$ \\
\hline GO:0022898 & $\mathrm{BP}$ & regulation of transmembrane transporter activity & 0.000107867 \\
\hline GO:0050806 & $\mathrm{BP}$ & positive regulation of synaptic transmission & 0.0001125 \\
\hline GO:0007411 & $\mathrm{BP}$ & axon guidance & 0.00012669 \\
\hline GO:0097485 & $\mathrm{BP}$ & neuron projection guidance & 0.000129216 \\
\hline GO:0032409 & $\mathrm{BP}$ & regulation of transporter activity & 0.000145222 \\
\hline GO:2001257 & $\mathrm{BP}$ & regulation of cation channel activity & 0.000147536 \\
\hline GO:0021953 & $\mathrm{BP}$ & central nervous system neuron differentiation & 0.000167914 \\
\hline GO:0007215 & $\mathrm{BP}$ & glutamate receptor signaling pathway & 0.000185499 \\
\hline GO:0050804 & $\mathrm{BP}$ & modulation of chemical synaptic transmission & 0.000220097 \\
\hline GO:0099177 & $\mathrm{BP}$ & regulation of trans-synaptic signaling & 0.000223196 \\
\hline GO:0007409 & $\mathrm{BP}$ & axonogenesis & 0.000338258 \\
\hline GO:0031103 & $\mathrm{BP}$ & axon regeneration & 0.000408548 \\
\hline GO:0031915 & $\mathrm{BP}$ & positive regulation of synaptic plasticity & 0.000427816 \\
\hline GO:0045665 & $\mathrm{BP}$ & negative regulation of neuron differentiation & 0.000435771 \\
\hline GO:0008076 & $\mathrm{CC}$ & voltage-gated potassium channel complex & 3.97E-06 \\
\hline GO:0034705 & $\mathrm{CC}$ & potassium channel complex & $6.45 \mathrm{E}-06$ \\
\hline GO:0034702 & $\mathrm{CC}$ & ion channel complex & $1.73 \mathrm{E}-05$ \\
\hline GO:0097060 & $\mathrm{CC}$ & synaptic membrane & $2.14 \mathrm{E}-05$ \\
\hline GO:1902495 & $\mathrm{CC}$ & transmembrane transporter complex & 2.77E-05 \\
\hline Go:0034703 & $\mathrm{CC}$ & cation channel complex & $2.98 \mathrm{E}-05$ \\
\hline GO:1990351 & $\mathrm{CC}$ & transporter complex & $3.23 \mathrm{E}-05$ \\
\hline GO:0001725 & $\mathrm{CC}$ & stress fiber & $3.39 \mathrm{E}-05$ \\
\hline GO:0097517 & $\mathrm{CC}$ & contractile actin filament bundle & 3.39E-05 \\
\hline G0:0099056 & $\mathrm{CC}$ & integral component of presynaptic membrane & 4.75E-05 \\
\hline
\end{tabular}




\begin{tabular}{|c|c|c|c|}
\hline G0:0032432 & $\mathrm{CC}$ & actin filament bundle & $5.29 \mathrm{E}-05$ \\
\hline GO:0099699 & $\mathrm{CC}$ & integral component of synaptic membrane & $5.95 \mathrm{E}-05$ \\
\hline GO:0042641 & CC & actomyosin & $6.48 \mathrm{E}-05$ \\
\hline GO:0098889 & $\mathrm{CC}$ & intrinsic component of presynaptic membrane & 7.50E-05 \\
\hline GO:0042734 & CC & presynaptic membrane & 7.81E-05 \\
\hline GO:0099240 & $\mathrm{CC}$ & intrinsic component of synaptic membrane & $8.52 \mathrm{E}-05$ \\
\hline GO:0045211 & $\mathrm{CC}$ & postsynaptic membrane & 0.000245873 \\
\hline GO:0099055 & $\mathrm{CC}$ & integral component of postsynaptic membrane & 0.00029622 \\
\hline GO:0043679 & $\mathrm{CC}$ & axon terminus & 0.000315999 \\
\hline GO:0044224 & $\mathrm{CC}$ & juxtaparanode region of axon & 0.000326672 \\
\hline GO:0005244 & MF & voltage-gated ion channel activity & 0.000251866 \\
\hline GO:0022832 & MF & voltage-gated channel activity & 0.000251866 \\
\hline GO:0022839 & MF & ion gated channel activity & 0.000380513 \\
\hline GO:0005267 & MF & potassium channel activity & 0.000431406 \\
\hline GO:0022836 & MF & gated channel activity & 0.000438171 \\
\hline GO:0016247 & MF & channel regulator activity & 0.000780857 \\
\hline GO:0005005 & MF & transmembrane-ephrin receptor activity & 0.001077167 \\
\hline GO:0015079 & MF & potassium ion transmembrane transporter activity & 0.001128962 \\
\hline GO:0005216 & MF & ion channel activity & 0.001198503 \\
\hline GO:0005003 & MF & ephrin receptor activity & 0.001349397 \\
\hline GO:0022838 & MF & substrate-specific channel activity & 0.001386177 \\
\hline GO:0015267 & MF & channel activity & 0.001911488 \\
\hline GO:0022803 & MF & passive transmembrane transporter activity & 0.0019327 \\
\hline GO:0005249 & MF & voltage-gated potassium channel activity & 0.00201551 \\
\hline GO:0005261 & MF & cation channel activity & 0.002197599 \\
\hline GO:0005251 & MF & delayed rectifier potassium channel activity & 0.004060627 \\
\hline GO:0015077 & MF & monovalent inorganic cation transmembrane transporter activity & 0.004749564 \\
\hline GO:0099106 & MF & ion channel regulator activity & 0.004767743 \\
\hline GO:0044325 & MF & ion channel binding & 0.005473012 \\
\hline GO:0022843 & MF & voltage-gated cation channel activity & 0.007951576 \\
\hline
\end{tabular}

BP: Biological processes; CC: Cellular components; MF: Molecular functions. 
only the top 20 entries with $\mathrm{P}$ values of $\mathrm{BP}, \mathrm{CC}$ and MF each are shown in the table. The rest are detailed in supplementary files S4.

\section{Table 3 KEGG enrichment results}

\begin{tabular}{|llll|}
\hline ID & Description & pvalue & gene \\
\hline hsa00532 & $\begin{array}{l}\text { Glycosaminoglycan biosynthesis - chondroitin } \\
\text { sulfate / dermatan sulfate }\end{array}$ & 0.001186412 & CHST13/CHST15 \\
\hline hsa04360 & Axon guidance & 0.011064786 & DCC/EPHA4/EPHB2 \\
\hline hsa04727 & GABAergic synapse & 0.021958488 & ABAT/GNG7 \\
\hline hsa05132 & Salmonella infection & 0.02545973 & AHNAK/RAB9B/TUBB2A \\
\hline
\end{tabular}

Table 4 GSEA enrichment results

\begin{tabular}{|lllll|}
\hline gene & Description & enrichmentScore & NES & pvalue \\
\hline EPHA4 & KEGG_AXON_GUIDANCE & 0.441773261 & 1.445925667 & 0.000999001 \\
\hline NCAM1 & KEGG_CELL_ADHESION_MOLECULES_CAMS & 0.495930892 & 1.550579226 & 0.000999001 \\
\hline NRXN1 & KEGG_CELL_ADHESION_MOLECULES_CAMS & 0.460731645 & 1.678777334 & 0.001046025 \\
\hline
\end{tabular}

only the pathways including the gene are shown in the table. The rest are detailed in supplementary files S5.

\section{Figures}


(A)

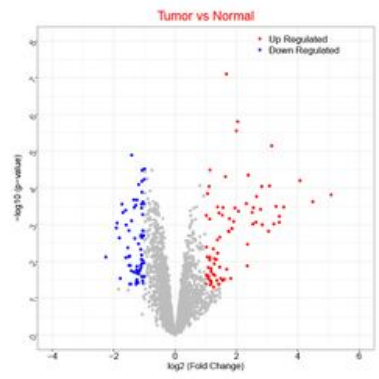

(D)

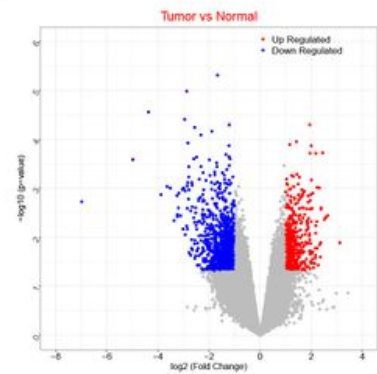

(G)

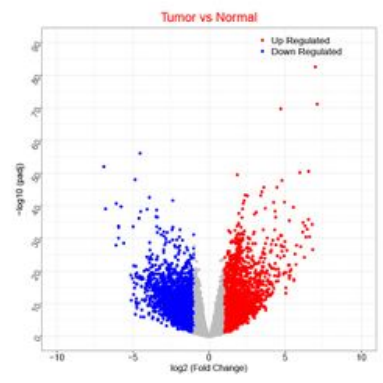

(J)

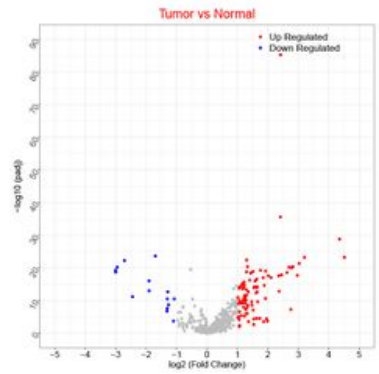

(B)

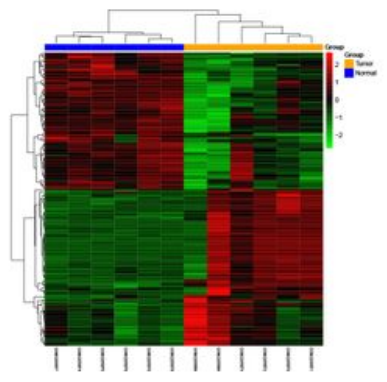

(E)

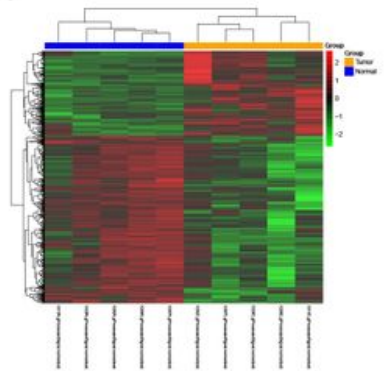

(H)

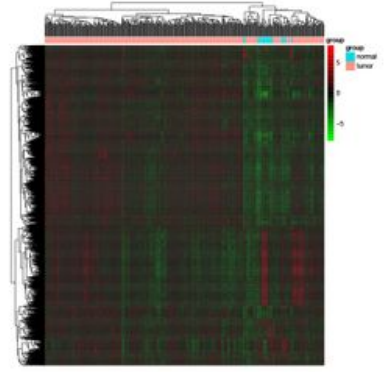

(K)

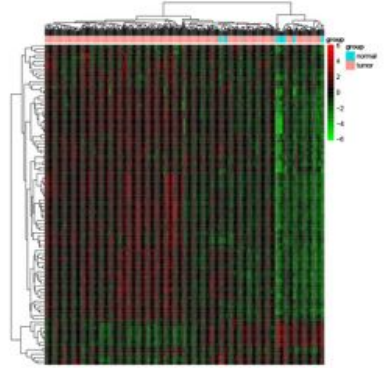

(C)

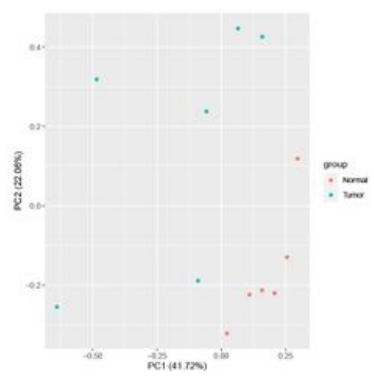

(F)

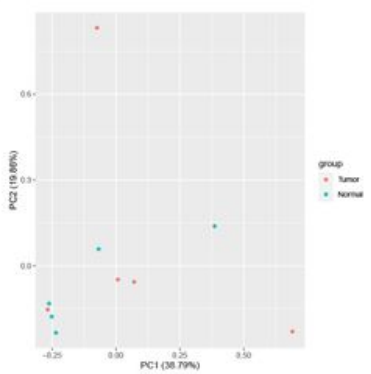

(I)

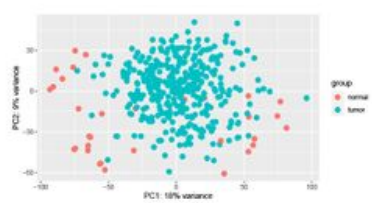

(L)

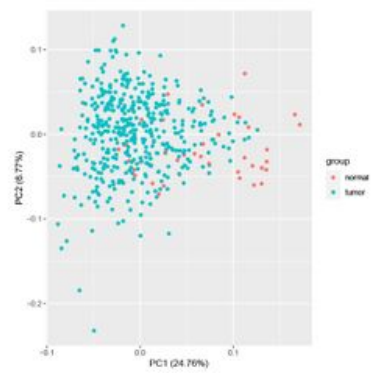

Figure 1

Differentially expressed genes. Volcano plot, heatmap and PCA diagram of DEcircRNAs from GSE83521 (A-C), DEcircRNAs from GSE100170 (D-F), DEmRNAs from TCGA database (G-I) and DEmiRNAs from TCGA database $(\mathrm{J}-\mathrm{L})$. 
(A)

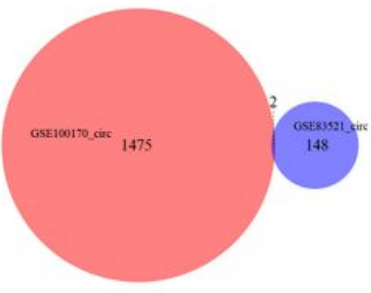

(D)

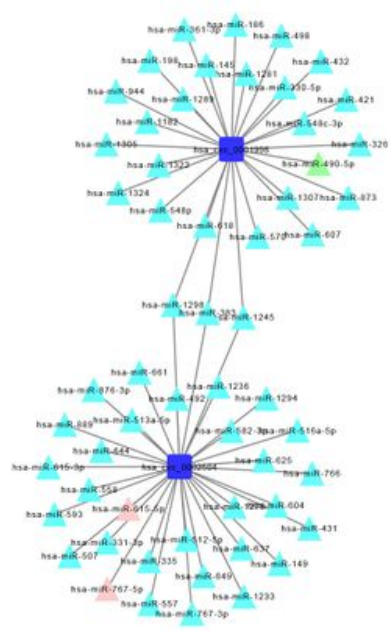

(G)

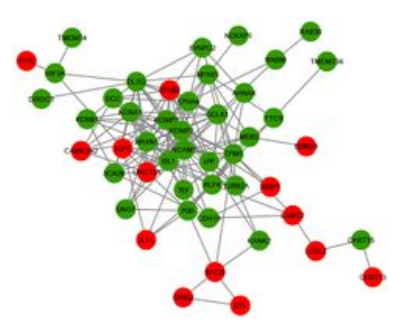

(B)

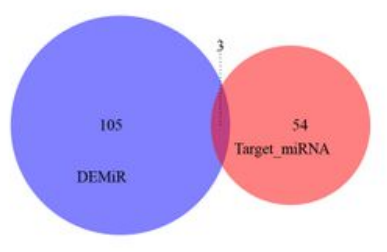

(E)

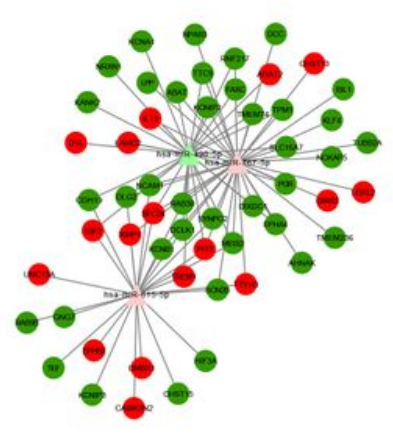

( $\mathrm{H})$

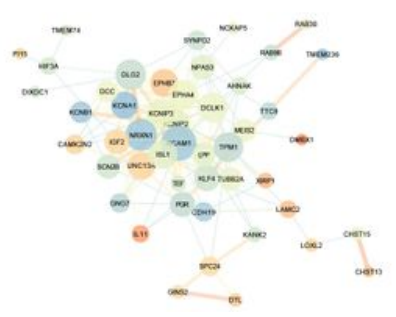

(C)

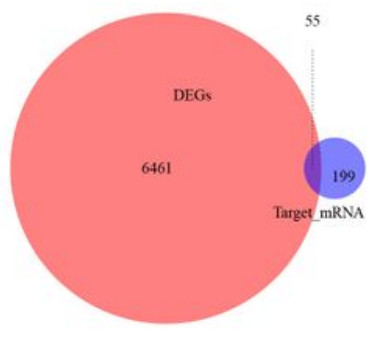

(F)

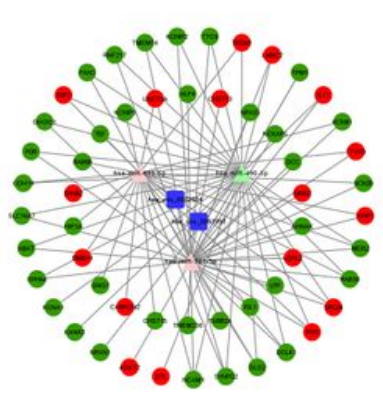

(I)

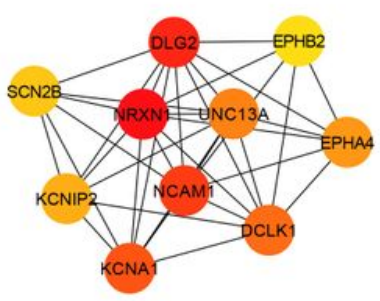

Figure 2

ceRNA and PPI interaction networks. (A-C) Venn diagram of intersected circRNAs, miRNAs and mRNAs. (D-F) Regulatory networks of circRNA-miRNA, miRNA-mRNA and ceRNA: The blue square represents the DEcircRNAs, triangle, the DEmiRNAs, green circle represents the down-regulated DEmRNAs, while red circle represents the upregulated DemRNAs. (G) interaction network of PPI. (H) PPI interaction network analyzer visualization: dot size represents degree value size, red represents up-regulated while blue represents down-regulated, edge thickness stands for combined score. (I) interaction network of Hub genes. 
(A)

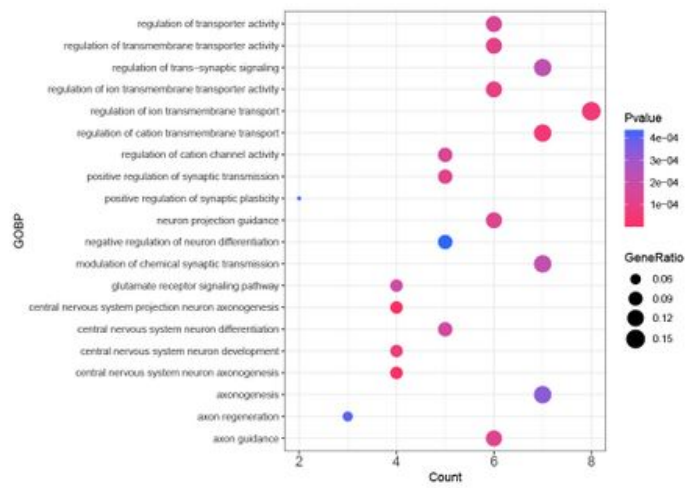

(C)

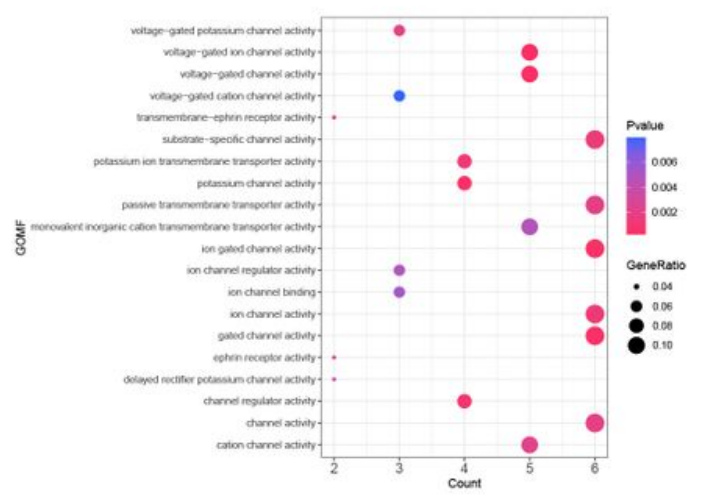

(E)

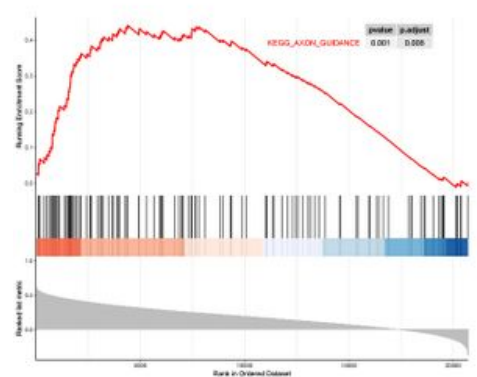

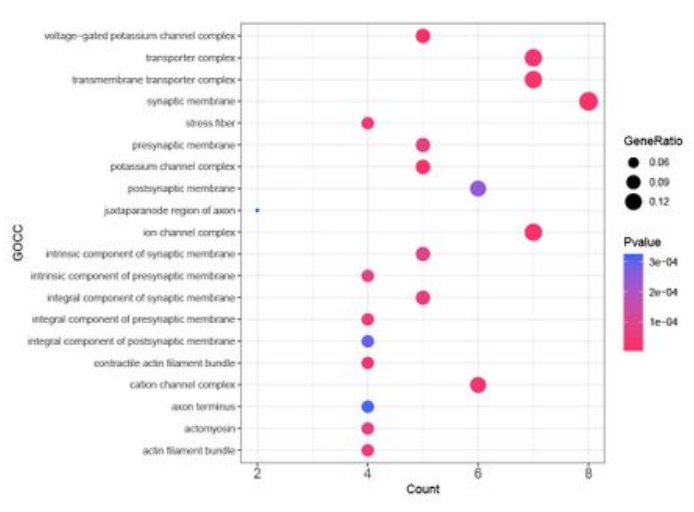

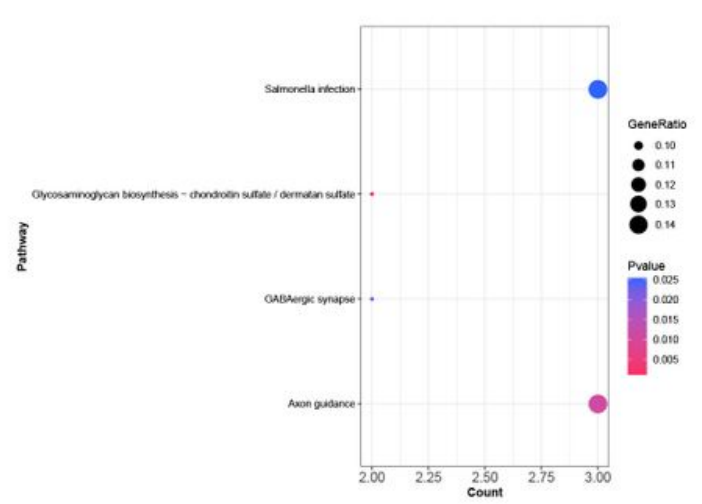

(D)

(B)

$(\mathrm{G})$

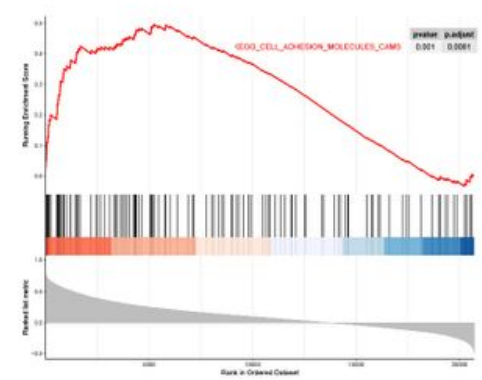

\section{Figure 3}

Function enrichment analysis. (A-D)Bubble diagrams of overlapping genes in GO KKEGG enrichment analysis: GOBP, GOCC, GOMF and KEGG pathway. (E-G) GSEA enrichment analysis of EPHA4, NCAM1 and NRXN1. BP: biological processes, CC: cellular composition, MF: molecular function. 
(A)

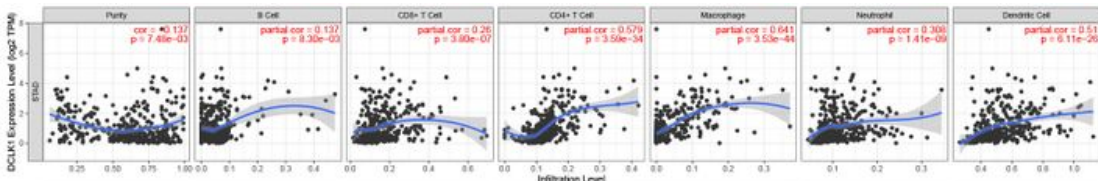

(B)

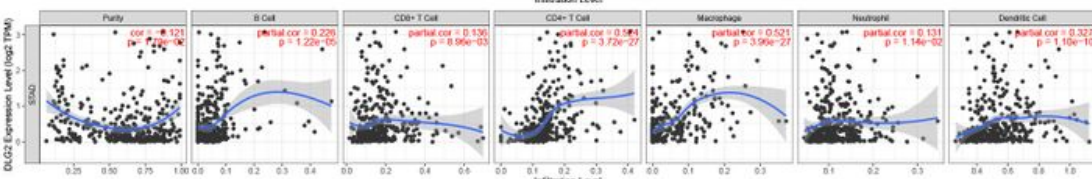

(C)

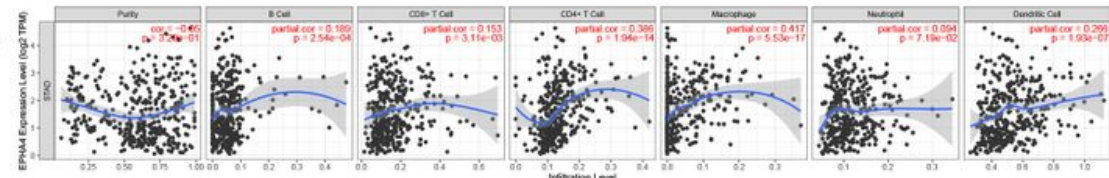

(D)

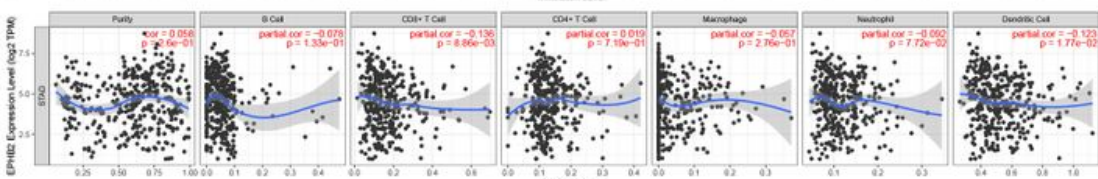

(E)

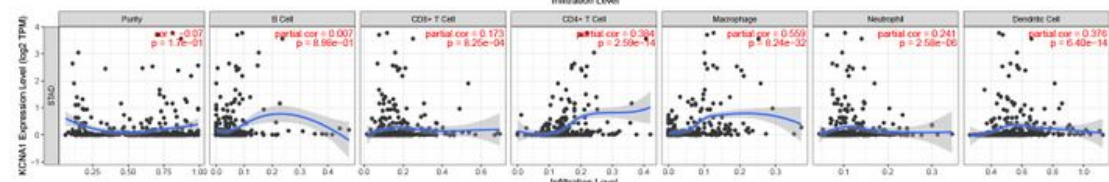

(F)

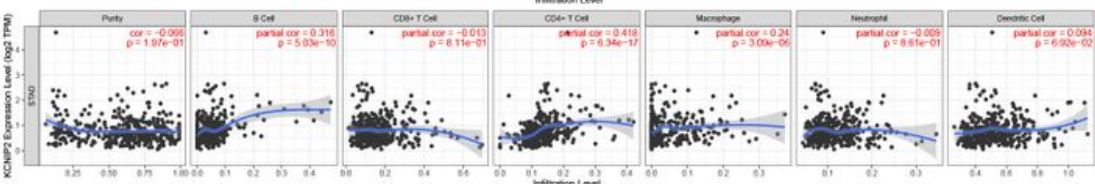

(G)

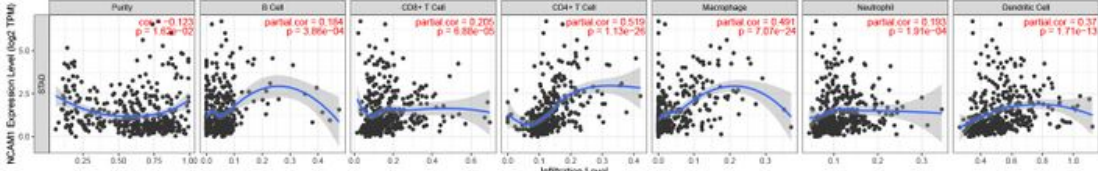

(H)

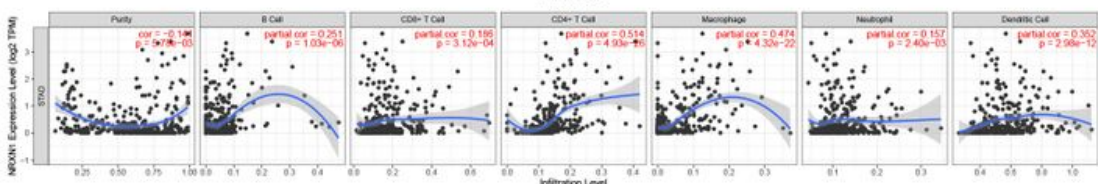

(I)

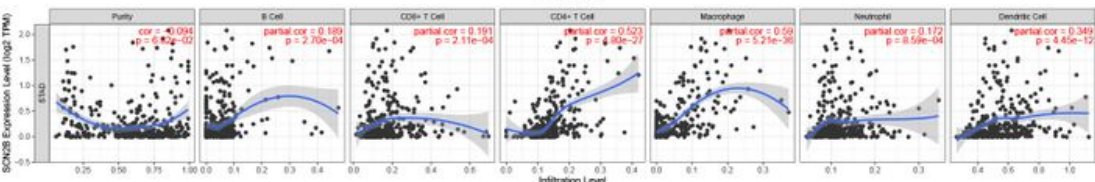

(J)

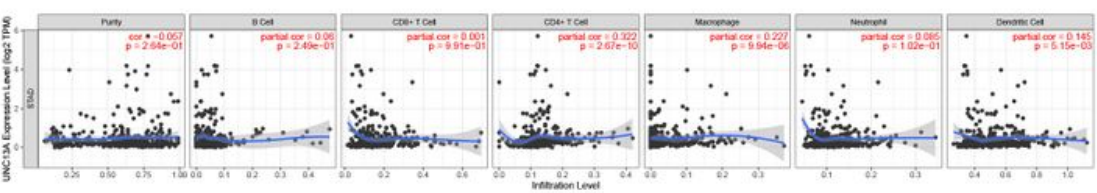

Figure 4

Immune infiltration analysis of hub genes. 

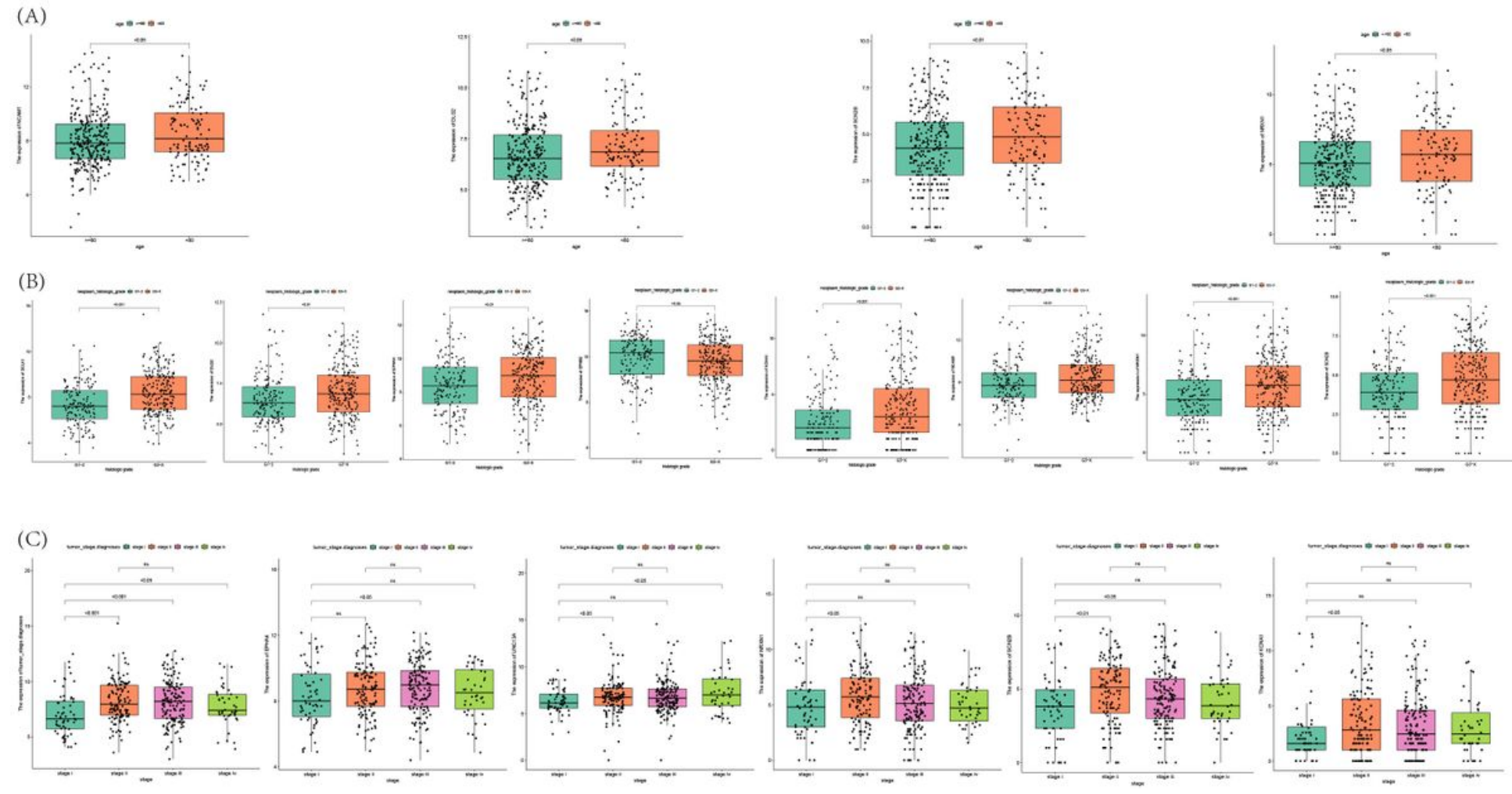

Figure 5

Clinical correlation analysis of hub genes. (A) age-related (B) histologic grade-related (C) tumor stage-ralated.

(A)

(B)

(C)
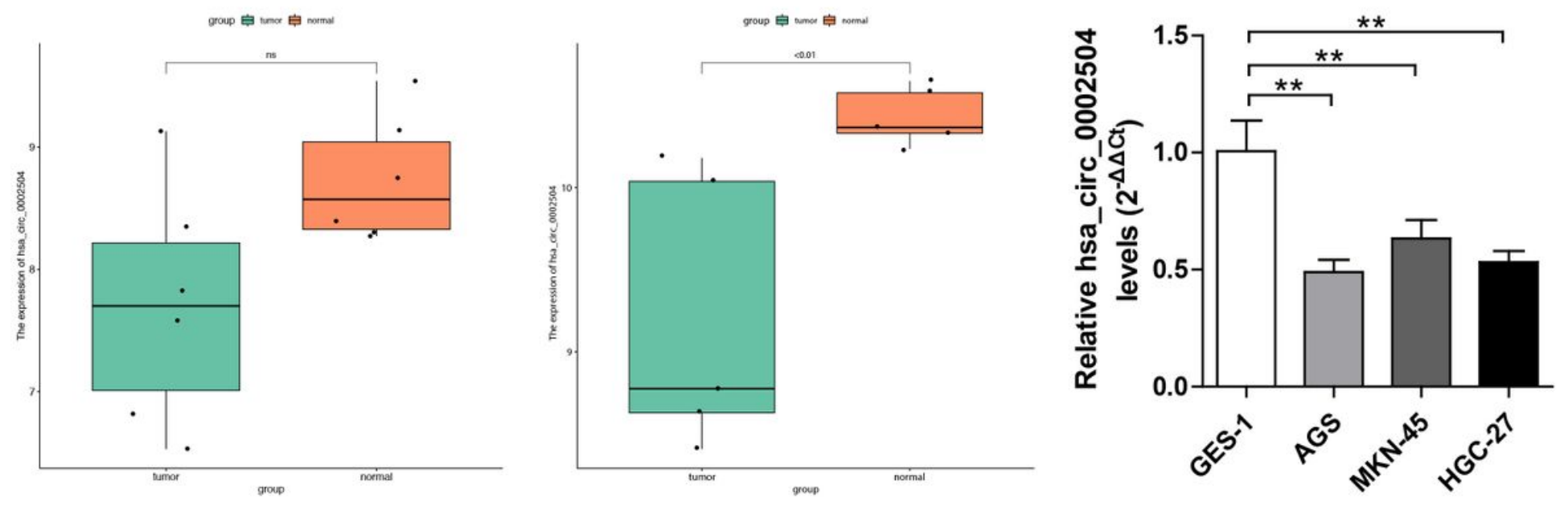

Figure 6

Expression of hsa_circ_0002504 in GSE83521 (A), GSE100170 (B) and gastric cells (C).

\section{Supplementary Files}


This is a list of supplementary files associated with this preprint. Click to download.

- supplementalfiles.rar 